\title{
The impact of high-frequency magnetic stimulation of peripheral nerves: muscle hardness, venous blood flow, and motor function of upper extremity in healthy subjects
}

\author{
Yoshihiko Okudera ${ }^{1}$, Toshiki Matsunaga ${ }^{2}$, Mineyoshi Sato ${ }^{2}$, Satoaki Chida ${ }^{2}$, Kazutoshi Hatakeyama ${ }^{2}$, \\ Motoyuki Watanabe ${ }^{2}$, and Yoichi Shimada ${ }^{1}$ \\ ${ }^{1}$ Department of Orthopedic Surgery, Akita University Graduate School of Medicine and ${ }^{2}$ Department of Rehabilitation Medicine, Akita \\ University Hospital, Akita 010-8543, Japan
}

(Received 2 December 2014; and accepted 12 December 2014)

\begin{abstract}
The purpose of this study was to investigate the impact of high-frequency peripheral nerve magnetic stimulation on the upper limb function. Twenty-five healthy adults (16 men and 9 women) participated in this study. The radial nerve of the non-dominant hand was stimulated by high-frequency magnetic stimulation device. A total of 600 impulses were applied at a frequency of $20 \mathrm{~Hz}$ and intensity of 1.2 resting motor threshold (rMT). At three time points (before, immediately after, and $15 \mathrm{~min}$ after stimulation), muscle hardness of the extensor digitorum muscle on the stimulated side was measured using a mechanical tissue hardness meter and a shear wave imaging device, cephalic venous blood flow on the stimulated side was measured using an ultrasound system, and the Box and Block test (BBT) was performed. Mechanical tissue hardness results did not show any significant differences between before, immediately after, and 15 min after stimulation. Measurements via shear wave imaging showed that muscle hardness significantly decreased both immediately and $15 \mathrm{~min}$ after stimulation compared to before stimulation $(P<0.05)$. Peripheral venous blood flow and BBT score significantly increased both immediately and 15 min after stimulation compared to before stimulation $(P<0.01)$. High-frequency peripheral nerve magnetic stimulation can achieve effects similar to electrical stimulation in a less invasive manner, and may therefore become an important element in next-generation rehabilitation.
\end{abstract}

In a pathological state in which the muscle cannot contract voluntarily due to upper motor neuron disorders such as stroke and spinal cord injury, it is possible to contract the paralyzed muscle by directly applying electrical stimulation to the lower motor neuron and its innervating muscles (21). Functional electrical stimulation (FES) can be applied to such motor paralysis; specifically, using an FES system, targeted movements can be recovered by applying

Address correspondence to: Yoshihiko Okudera, MD, Department of Orthopedic Surgery, Akita University Graduate School of Medicine, 1-1-1 Hondo, Akita 0108543, Japan

Tel: +81-18-884-6148, Fax: +81-18-884-6354

E-mail: one.punc@gmail.com programmed movement stimuli to the paralyzed limb via multiple stimulation electrodes (22). Moreover, a recent report using fMRI demonstrated afferent effects in the motor cortex after FES (20), and FES is therefore anticipated for future development in the field of rehabilitation.

The electrodes utilized in electrical stimulation are generally classified into surface electrodes or implanted electrodes. Some of the disadvantages of surface electrodes include: they induce pain and have risk of burn due to Joules heat caused by a concentration of current that is dependent on the non-uniform distribution of electrode contact impedance; they can only stimulate the shallow muscle layer and cannot selectively apply stimuli; and they require time for attachment and detachment (9). On 
the other hand, while implanted electrodes have the advantage over surface electrodes of having the capability to selectively stimulate the deep muscle layer, they have several disadvantages including a necessity for an invasive surgical operation for insertion, risk of infection, and breakage of electrodes (9).

Magnetic stimulation is a method in which the nerve is stimulated through an eddy current arising from a magnetic field that occurs perpendicular to the plane of the stimulation coil when current passes through the coil (2). Unlike electrical stimulation, magnetic stimulation is a non-contact method with very few occurrences of pain caused by skin impedance, and has an advantage of being able to stimulate deep tissue areas noninvasively (14).

There have been many clinical reports on the improvement in motor function of a paralyzed limb by transcranial magnetic stimulation $(13,24)$. Transcranial magnetic stimulation in stroke patients with hemiplegia leads to a decrease in excitability of the unaffected motor cortex with low-frequency stimulation, and an enhanced motor training effect on the paralyzed limb through the activation of the pyramidal tract function and augmentation of cerebral cortex plasticity with high-frequency stimulation (24). Magnetic stimulation of the peripheral nerves has been primarily performed at high frequencies, and has demonstrated preventative effects against muscle atrophy in animals (18). In humans, effects at the central nervous system level have been reported with high-frequency peripheral nerve magnetic stimulation, which was shown to induce the activation of frontal and parietal lobes of magnetic stimulation on motor function in humans (23). However, in contrast to the clinical reports on transcranial magnetic stimulation, no reports to our knowledge have investigated the effects of high-frequency peripheral nerve magnetic stimulation on motor function in humans.

The purpose of this study is to investigate the impact of high-frequency magnetic stimulation of peripheral nerves on the upper limb function.

\section{MATERIALS AND METHODS}

Subjects. We investigated twenty-five healthy adults (16 men and 9 women). There were 24 right-handed and 1 left-handed participants, and the mean age was 28.8 years, mean height was $168.3 \mathrm{~cm}$, and mean weight was $65.2 \mathrm{~kg}$. This study was approved by the Ethics Committee of Akita University. Written consent was obtained from all participants prior to the study.
Interventions. Participants were in a sitting position with $90^{\circ}$ elbow joint flexion and pronated forearm. Using a high-frequency magnetic stimulator (MagPro R100; MagVenture Inc., Atlanta, GA, USA), the radial nerve of the non-dominant hand was repetitively stimulated. The conditions of the stimuli were such that a total of 600 continuous impulses were applied in $55 \mathrm{~s}$ at a frequency of $20 \mathrm{~Hz}$ and intensity of $1.2 \mathrm{rMT}$. Stimulation was verified by assessing the motor-evoked potential (MEP) from the extensor digitorum muscle on the stimulated side.

Magnetic stimulation was applied in accordance with safety guidelines, and the safety of the participants was ensured by immediately discontinuing the stimuli if they had complaints, such as experiencing unpleasant feelings during stimulation (17).

\section{Outcome assessment}

All measurements were performed on the non-dominant hand.

Muscle hardness measurement by a mechanical muscle hardness meter. Using a mechanical tissue hardness meter (PEK-1; Imoto Machinery, Co., Ltd., Kyoto, Japan), hardness of the extensor digitorum muscle on the stimulated side was measured before, immediately after, and $15 \mathrm{~min}$ after magnetic stimulation. Participants were in a sitting position for measurements, and the table was positioned such that its height was at the olecranon when the upper limb was extended downward. The mechanical tissue hardness meter is pressed onto the measurement site on the participant by the examiner to measure the pressure difference between the periphery and section to be measured.

Muscle hardness measurement by ultrasonic shear wave imaging. Using a shear wave imaging ultrasound system (ACUSON S3000; Siemens Japan K.K., Tokyo, Japan), muscle hardness of the extensor digitorum muscle on the stimulated side was measured before, immediately after, and $15 \mathrm{~min}$ after magnetic stimulation (Fig. 1). Participants were in a sitting position for measurements, and the table was positioned such that its height was at the olecranon when the upper limb was extended downward.

Shear wave imaging applies the concept that propagating velocity shows a positive correlation with Young's modulus, which represents the hardness of the object, and visualizes the hardness of an object by sending a transverse elastic wave, or shear wave, to the inner part of the tissue and measuring the propagating velocity. Since images can be captured with the probe in a fixed position on the body sur- 
face, there is minimal operator-dependence and good reproducibility, and a stable elastography can be obtained through this method (12). Taking advantage of these particular features of shear wave imaging, its clinical application has been progressing in liver fibrosis diagnosis, an important factor in chronic liver diseases (5), and in breast disorders since tissue hardness is a crucial element in this field as well $(1,25)$.

Measurement of cephalic venous blood flow on the stimulated side with an ultrasound. Using an ultrasound system (ACUSON S3000; Siemens Japan K.K.), cephalic venous blood flow on the stimulated side was measured before, immediately after, and 15 min after magnetic stimulation (Fig. 2). Participants were in a sitting position for measurements, and the table was positioned such that its height was

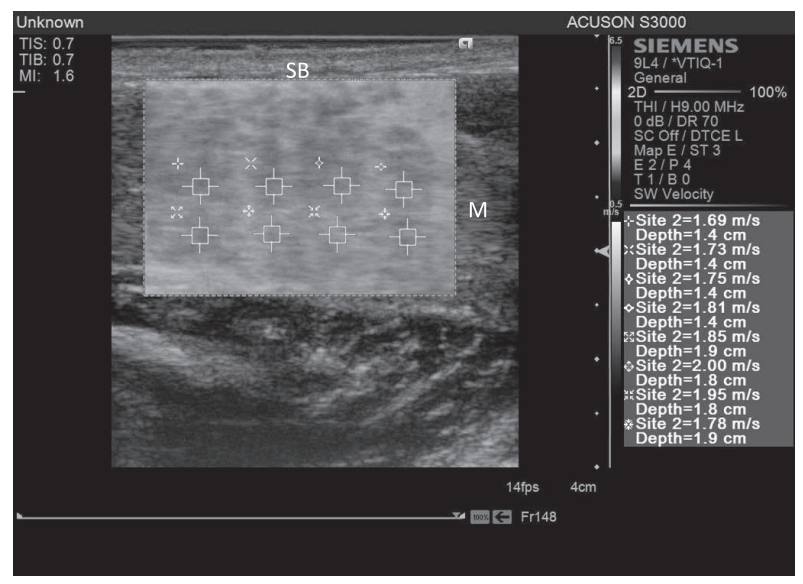

Fig. 1 Visualization of ultrasonic shear wave imaging. This technique visualizes and digitizes hardness by sending shear waves to the inner area of tissues and measuring the resulting propagating velocity. Manual compression is not required for measurement. SB: subcutaneous tissue, M: common digital extensor muscle. at the olecranon when the upper limb was extended downward. Venous blood flow was automatically calculated by visualizing the maximum diameter of the blood vessel of interest and by analyzing the waveform. To enhance the reproducibility, the position of the probe for the measurement before magnetic stimulation was marked on the skin.

Box and block test (BBT). Using the Box and Block test (BBT; Reha-Stim Medtec Gmbh \& Co. Kg, Berlin, Germany), the number of blocks transported over the partition in a 60 -s period with maximum effort was scored before, immediately after, and $15 \mathrm{~min}$ after stimulation. A 15 -s trial period was provided prior to the actual test. Blocks that were tossed without the participant's fingers crossing the partition and blocks that bounced out of the box without crossing the partition were excluded. If two blocks were transported at the same time, it was counted as one. The mean score has previously been determined by age group, and this test has been used primarily to evaluate gross finger dexterity (10). Participants were in a sitting position for measurements, and the table was positioned such that its height was at the olecranon when the upper limb was extended downward.

Data analysis. The Bonferroni method was used to validate statistical significances between the three groups (before, immediately after, and $15 \mathrm{~min}$ after stimulation) in each of the following measurements: muscle hardness determined by mechanical muscle hardness meter and by ultrasonic shear wave imaging, cephalic venous blood flow on the stimulated side determined by ultrasound, and score from the BBT. $P<0.001$ was considered statistically significant.

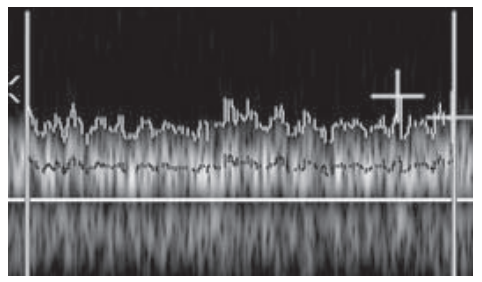

a Before stimulation
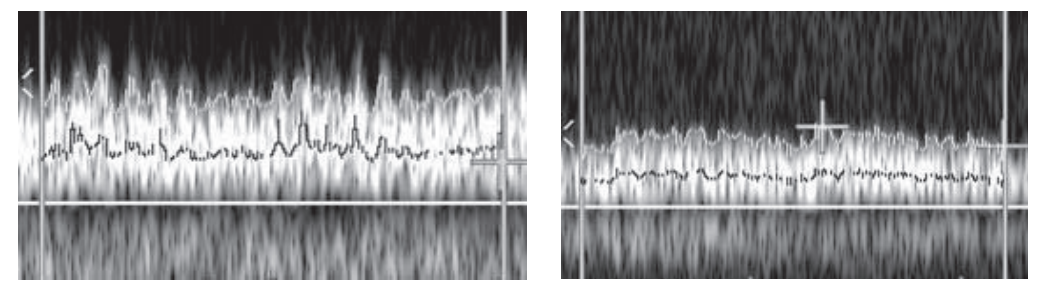

b Immediately after stimulation

\section{c 15 min after stimulation}

Fig. 2 Image of cephalic venous blood flow measured on the stimulated side by ultrasound. a: Blood flow before stimulation, b: Blood flow immediately after stimulation, and c: Blood flow $15 \mathrm{~min}$ after stimulation. Increased blood flow was observed after repetitive magnetic stimulation. 


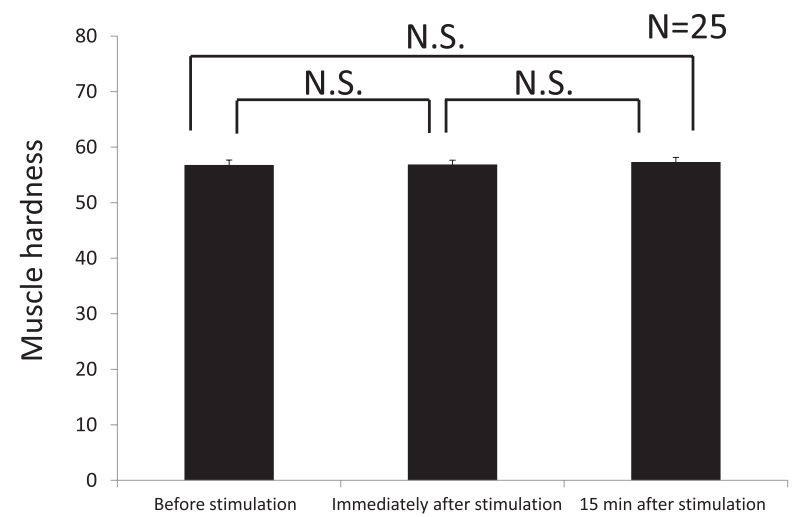

Fig. 3 Muscle hardness measured by mechanical tissue hardness meter. Significant differences were not observed between the three groups.

\section{RESULTS}

Changes in muscle hardness before and after magnetic stimulation

Muscle hardness measured by the mechanical tissue hardness meter was: $54 \pm 4.8$ (mean \pm SD) before stimulation, $54 \pm 4.6$ immediately after stimulation, and $55 \pm 4.915 \mathrm{~min}$ after stimulation, indicating that significant differences were not observed between the groups (Fig. 3).

Muscle hardness determined by shear wave imaging was: $2.5 \pm 0.36$ (mean $\pm \mathrm{SD}$ ) $\mathrm{m} / \mathrm{s}$ before stimulation, $2.0 \pm 0.29 \mathrm{~m} / \mathrm{s}$ immediately after stimulation, and $2.0 \pm 0.31 \mathrm{~m} / \mathrm{s} 15 \mathrm{~min}$ after stimulation. This demonstrated that muscle hardness significantly decreased both immediately after and $15 \mathrm{~min}$ after stimulation compared to before stimulation [before stimulation vs. immediately after stimulation: $95 \%$ confidence interval $0.115-0.478(P<0.001)$; before stimulation vs. $15 \mathrm{~min}$ after stimulation: $95 \%$ confidence interval $0.153-0.557(P<0.001)]$. Although decreased muscle hardness continued 15 min after stimulation, there was no significant difference between immediately after and $15 \mathrm{~min}$ after stimulation [95\% confidence interval -0.069 to 0.186 ] (Fig. 4).

\section{Changes in peripheral venous blood flow}

Peripheral venous blood flow was $4.5 \pm 0.74 \mathrm{~mL} / \mathrm{min}$ (mean $\pm \mathrm{SD}$ ) before stimulation, $7.0 \pm 1.0 \mathrm{~mL} / \mathrm{min}$ immediately after stimulation, and $8.6 \pm 1.3 \mathrm{~mL} / \mathrm{min}$ 15 min after stimulation, indicating a significant increase both immediately and 15 min after stimulation compared to before stimulation [before stimulation vs. immediately after stimulation: $95 \%$ confidence interval -0.004 to $-0.001(P<0.001)$, before stimulation vs. $15 \mathrm{~min}$ after stimulation: $95 \%$ confidence

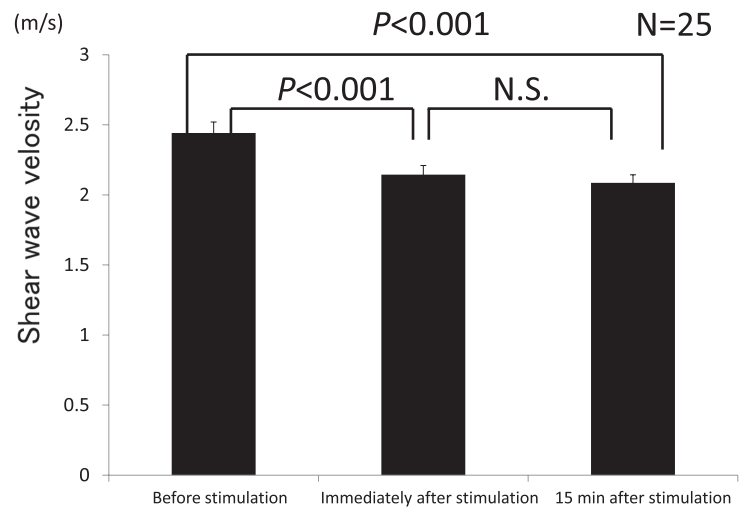

Fig. 4 Muscle hardness measured by ultrasonic shear wave imaging. Muscle hardness measured by ultrasonic shear wave significantly decreased both immediately after and 15 min after stimulation compared to before stimulation [before stimulation vs. immediately after stimulation: $95 \%$ confidence interval $0.115-0.478(P<0.001)$; before stimulation vs. $15 \mathrm{~min}$ after stimulation: $95 \%$ confidence interval 0.153 $0.557(P<0.001)]$.

interval -0.006 to $-0.002(P<0.001)]$. Although increased blood flow continued 15 min after stimulation, there was no significant difference between immediately after and $15 \mathrm{~min}$ after stimulation [95\% confidence interval -0.003 to 0.000 ] (Fig. 5).

\section{Changes in BBT score}

BBT score was $67 \pm 1.4$ (mean $\pm \mathrm{SD}$ ) before stimulation, $74 \pm 1.4$ immediately after stimulation, and $76 \pm 1.415 \mathrm{~min}$ after stimulation, indicating a significant increase both immediately after and $15 \mathrm{~min}$ after stimulation compared to before stimulation [before stimulation vs. immediately after stimulation: $95 \%$ confidence interval -9.034 to $-6.566(P<$ 0.001 ), before stimulation vs. 15 min after stimulation: $95 \%$ confidence interval -11.273 to -7.207 $(P<0.001)]$. Although the increased BBT score continued 15 min after stimulation, a significant difference was not observed between immediately after and $15 \mathrm{~min}$ after stimulation [95\% confidence interval -3.115 to 0.235 ] (Fig. 6).

\section{DISCUSSION}

In the present study, we applied repetitive magnetic stimuli to peripheral nerves and investigated their effects on the upper limb function in healthy individuals. Several research groups have reported that electrical stimulation can provide a more effective rehabilitation when combined with conventional physiotherapy, and it has become indispensible in modern-day rehabilitation $(11,18,21)$. Since mag- 


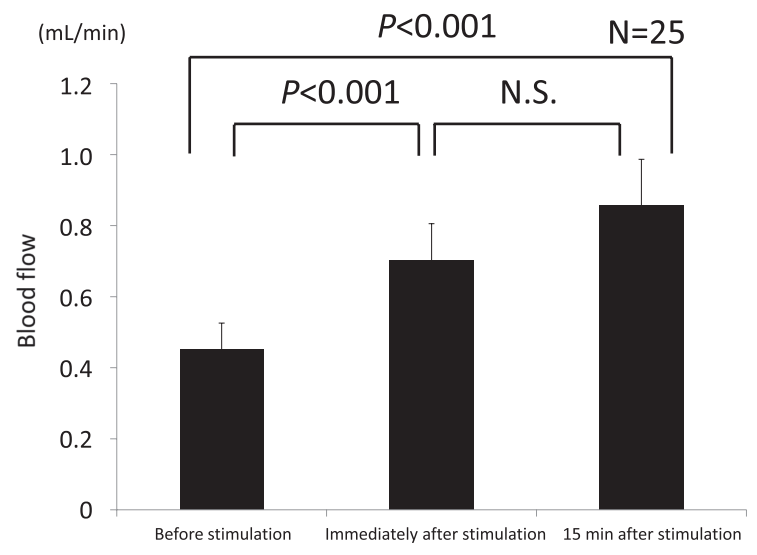

Fig. 5 Peripheral venous blood flow. Peripheral venous blood flow significantly increased both immediately after and $15 \mathrm{~min}$ after stimulation compared to before stimulation [before stimulation vs. immediately after stimulation: 95\% confidence interval -0.004 to $-0.001 \quad(P<0.001)$; before stimulation vs. $15 \mathrm{~min}$ after stimulation: $95 \%$ confidence interval -0.006 to $-0.002(P<0.001)$ ].

netic stimulation can stimulate deep tissue areas less invasively than electrical stimulation (14), if it is proven to have similar or greater effects compared to electrical stimulation, it may become a novel rehabilitation technique.

Changes in muscle hardness with repetitive magnetic stimulation

Due to its simplicity and convenience, muscle hardness measurement via a mechanical muscle hardness meter has been utilized in various settings including studies on the correlation with muscle fatigue, association with muscle overuse in sports (15), and association with myofascial pain (8). In the present study, we did not observe changes in muscle hardness before and after magnetic stimulation with a mechanical muscle hardness meter.

In contrast, decreased muscle hardness was evident with shear wave imaging. The reason for this was considered to be due to the specific features of shear wave imaging that it can measure muscle hardness without passing through the skin or fat and that it does not require the tissue to be manually compressed. Consequently, there is minimal error between examiners, leading to measurements with greater accuracy. It is possible that the mechanical muscle hardness meter was not able to detect changes in muscle hardness as sensitively as shear wave imaging. The electrical stimulation of peripheral nerves activates group Ia afferent nerve fibers from stimulated muscle, group Ib afferent nerve fibers from the Golgi-tendon organ, and group II afferent

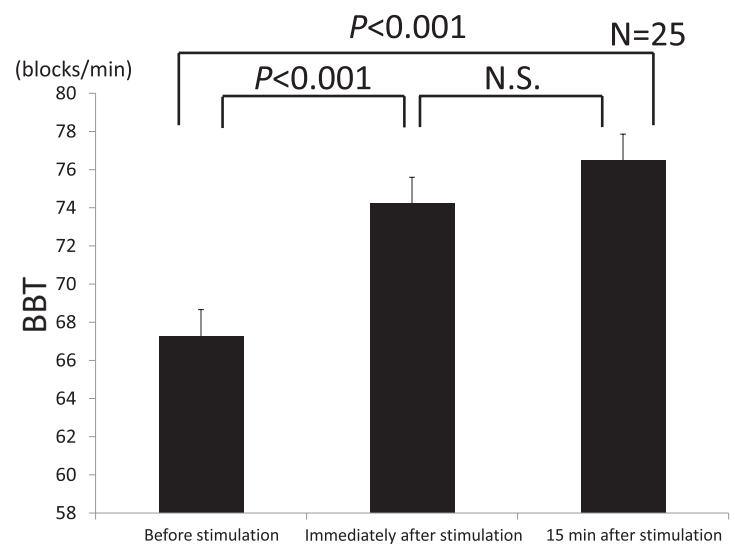

Fig. 6 BBT results. The number of transported blocks (score) in BBT significantly increased both immediately after and $15 \mathrm{~min}$ after stimulation compared to before stimulation [before stimulation vs. immediately after stimulation: 95\% confidence interval -9.034 to $-6.566(P<0.001)$; before stimulation vs. 15 min after stimulation: $95 \%$ confidence interval -11.273 to $-7.207(P<0.001)$ ].

nerve fibers from the skin, thereby decreasing muscle hardness (27). It is possible that high-frequency magnetic stimulation of peripheral nerves decreased muscle hardness via a similar mechanism.

Changes in peripheral venous blood flow due to repetitive magnetic stimulation

In the present study, peripheral venous blood flow on the stimulated side significantly increased after magnetic stimulation. To our knowledge, no previous studies have measured blood flow after magnetic stimulation of the peripheral nerves, and our study is the first to report this finding. Coles et al. (3) reported that, with electrical stimulation, venous return increases due to muscle contraction at the time of stimulation. It is plausible that a similar effect occurred also with magnetic stimulation.

Recently, there has been an increasing number of reports on deep venous thrombosis prophylaxis by increasing venous return via electrical stimulation of the peripheral nerves $(4,6,19,26)$. Since high-frequency peripheral nerve magnetic stimulation can be performed without direct contact, it is applicable for patients immobilized with a cast. Moreover, it may promote thromboprophylaxis less invasively compared to other methods as it does not induce pain.

\section{Changes in BBT score after repetitive magnetic stim- ulation}

BBT score significantly increased after stimulation, indicating an improvement in motor function. Since the actual test was performed after a 15 -s trial peri- 
od in accordance with the guideline, we believe that there is very little influence from learning effects (10). Koesler et al. (7) showed an improvement in upper limb coordination after electrical stimulation of peripheral nerves, and reported that corticospinal excitability also increases in healthy individuals after electrical stimulation of the peripheral nerves.

\section{Sustained effects of stimulation}

In the present study, decreased muscle hardness, increased blood flow, and improved motor function all continued even at $15 \mathrm{~min}$ after the completion of stimuli, demonstrating sustained effects of stimulation. There is a report that confirmed the sustained cortical excitability $15 \mathrm{~min}$ after peripheral nerve electrical stimulation with transcranial magnetic stimulation-elicited MEPs (16), and this particular finding was considered to be one of the reasons why the effects of stimulation were sustained in our study. In the future, it will be necessary to also investigate the duration of stimulation effects in each type of measurement.

\section{Study limitations}

The present study did not directly compare electrical and magnetic stimuli. In the future, a direct comparison between these two types of stimuli is necessary. Magnetic stimulation even to the peripheral nerves is contraindicated in individuals with pacemakers, implantable defibrillators, and metal implants, which are all common in elderly people. Therefore, individuals who are eligible for this type of stimulation in the clinical setting may be limited to some extent.

In conclusions, we investigated the effects of high-frequency magnetic stimulation of peripheral nerves on the upper limb function in healthy adults. After stimulation, we observed decreased muscle hardness through shear wave imaging, increased peripheral circulatory blood flow, and improved motor function. High-frequency peripheral nerve magnetic stimulation may achieve effects similar to electrical stimulation in a less-invasive manner, and may become a novel technique to be used in rehabilitation.

\section{REFERENCES}

1. Athanasiou A, Tardivon A, Tanter M, Sigal-Zafrani B, Bercoff J, Deffieux T, Gennisson JL, Fink M and Neuenschwander S (2010) Breast lesions: quantitative elastography with supersonic shear imaging - preliminary results. Radiology 256, 297-303.

2. Barker AT, Freeston IL, Jalinous R and Jarratt JA (1987) Magenetic stimulation of the human brain and peripheral nervous system: an introduction and the results of an initial clinical evaluation. Neurosurgery 20, 100-109.

3. Coles DR, Kidd BS and Moffat W (1957) Distensibility of blood vessels of the human calf determined by local application of subatmospheric pressures. J Appl Physiol 10, 461468.

4. Czyrny JJ, Kaplan RE, Wilding GE, Purdy CH and Hirsh J (2010) Electrical foot stimulation: a potential new method of deep venous thrombosis prophylaxis. Vascular 18, 20-27.

5. Ferraioli G, Parekh P, Levitov AB and Filice C (2014) Shear wave elastography for evaluation of liver fibrosis. $J$ Ultrasound Med 33, 197-203.

6. Hou LL, Yao LW, Niu QM, Xu L, Yu QH, Sun WQ, Yin PH and Li Q (2013) Preventive effect of electrical acupoint stimulation on lower-limb thrombosis: a prospective study of elderly patients after malignant gastrointestinal tumor surgery. Cancer Nurs 36, 139-144.

7. Koesler IB, Dafotakis M, Ameli M, Fink GR and Nowak DA (2009) Electrical somatosensory stimulation improves movement kinematics of the affected hand following stroke. $J$ Neurol Neurosurg Psychiatry 80, 614-619.

8. Kogo H and Kurosawa K (2010) Seeking the cause of myofascial pain syndrome by identifying which manual therapy is effective against muscle tenderness and stiffness. $J$ Phys Ther Sci 22, 173-176.

9. Mason JL and Mackay NA (1976) Pain sensations associated with electrocutaneous stimulation. IEEE Trans Biomed Eng 23, 405-409.

10. Mathiowetz V, Volland G, Kashman N and Weber K (1985) Adult norms for the Box and Block Test of manual dexterity. Am J Occup Ther 39, 386-391.

11. Matsunaga T, Shimada Y, Sato M, Chida S, Hatakeyama K and Misawa A (2007) Clinical experience of functional electrical stimulation (FES) for restoration of tetraplegic hand function. Akita J Med 34, 137-144.

12. Nightingale K, McAleavey S and Trahey G (2003) Shearwave generation using acoustic radiation force: in vivo and ex vivo results. Ultrasound Med Biol 29, 1715-1723.

13. Panouillères M, Neggers SF, Gutteling TP, Salemme R, van der Stigchel S, van der Geest JN, Frens MA and Pélisson D (2012) Transcranial magnetic stimulation and motor plasticity in human lateral cerebellum: dual effect on saccadic adaptation. Hum Brain Mapp 33, 1512-1525.

14. Polson MJ, Barker AT and Freeston IL (1982) Stimulation of nerve trunks with time-varying magnetic fields. Med Biol Eng Comput 20, 243-244.

15. Renström P and Johnson RJ (1985) Overuse injuries in sports. A review. Sports Med 2, 316-333.

16. Ridding MC, Brouwer B, Miles TS, Pitcher JB and Thompson PD (2000) Changes in muscle responses to stimulation of the motor cortex induced by peripheral nerve stimulation in human subjects. Exp Brain Res 131, 135-143.

17. Rossi S, Hallett M, Rossini PM, Pascual-Leone A and Safety of TMS Consensus Group (2009) Safety, ethical considerations, and application guidelines for the use of transcranial magnetic stimulation in clinical practice and research. Clin Neurophysiol 120, 2008-2039.

18. Sakuraba T, Shimada Y, Takahashi S, Matsunaga T, Itoi E and Kawatani M (2005) The effect of magnetic stimulation on unloaded soleus muscle of rat: changes in myosin heavy chain mRNA isoforms. Biomed Res (Tokyo) 26, 15-19.

19. Sandberg ML, Sandberg MK and Dahl J (2007) Blood flow changes in the trapezius muscle and overlying skin following transcutaneous electrical nerve stimulation. Phys Ther 87, 1047-1055. 
20. Sasaki K, Matsunaga T, Tomite T, Yoshikawa T and Shimada Y (2012) Effect of electrical stimulation therapy on upper extremity functional recovery and cerebral cortical changes in patients with chronic hemiplregia. Biomed Res (Tokyo) 33, 89-96.

21. Shimada Y, Sato K, Abe E, Kagaya H, Ebata K, Oba M and Sato M (1996) Clinical experience of functional electrical stimulation in complete paraplegia. Spinal Cord 34, 615-619.

22. Shimada Y, Sato K, Kagaya H, Konishi N, Miyamoto S and Matsunaga T (1996) Clinical use of percutaneous intramuscular electrodes for functional electrical stimulation. Arch Phys Med Rehabil 77, 1014-1018.

23. Struppler A, Binkofski F, Angerer B, Bernhardt M, Spiegel S, Drzezga A and Bartenstein P (2007) A fronto-parietal network is mediating improvement of motor function related to repetitive peripheral magnetic stimulation: A PET-H2O15 study. Neuroimage 36, 174-186.

24. Takeuchi N, Tada T, Toshima M, Chuma T, Matsuo Y and
Ikoma K (2008) Inhibition of the unaffected motor cortex by $1 \mathrm{~Hz}$ repetitive transcranical magnetic stimulation enhances motor performance and training effect of the paretic hand in patients with chronic stroke. J Rehabil Med 40, 298-303.

25. Tanter M, Bercoff J, Athanasiou A, Deffieux T, Gennisson JL, Montaldo G, Muller M, Tardivon A and Fink M (2008) Quantitative assessment of breast lesion viscoelasticity: initial clinical results using supersonic shear imaging. Ultrasound Med Biol 34, 1373-1386.

26. Warwick DJ, Shaikh A, Gadola S, Stokes M, Worsley P, Bain D, Tucker AT and Gadola SD (2013) Neuromuscular electrostimulation viathe common peroneal nerve promotes lower limb blood flow in a below-kneecast: A potential for thromboprophylaxis. Bone Joint Res 2, 179-185.

27. Wu CW, van Gelderen P, Hanakawa T, Yaseen $\mathrm{Z}$ and Cohen LG (2005) Enduring representational plasticity after somatosensory stimulation. Neuroimage $\mathbf{2 7}, 872-884$. 